\title{
Tarbes « Castrum Turba ou Tarba »
}

Place de Verdun

Jean-Michel Martin

\section{(2) OpenEdition}

Journals

Édition électronique

URL : http://journals.openedition.org/adlfi/10864

ISSN : 2114-0502

Éditeur

Ministère de la culture

Référence électronique

Jean-Michel Martin, «Tarbes « Castrum Turba ou Tarba » », ADLFI. Archéologie de la France -

Informations [En ligne], Midi-Pyrénées, mis en ligne le 01 mars 1997, consulté le 19 avril 2019. URL

http://journals.openedition.org/adlfi/10864

Ce document a été généré automatiquement le 19 avril 2019

(C) Ministère de la Culture et de la Communication, CNRS 


\title{
Tarbes « Castrum Turba ou Tarba »
}

\author{
Place de Verdun
}

Jean-Michel Martin

Date de l'opération : 1991 - 1992 (SP)

Inventeur(s) : Martin Jean-Michel

1 La ville de Tarbes n'avait jusqu'alors fait l'objet d'aucune fouille de grande envergure. Tout au plus s'agissait-il de suivi de travaux ou de sondages toujours trop réduits en surface et dont les résultats se limitaient à l'étude des niveaux gallo-romains au détriment des contextes médiévaux et post-médiévaux.

2 Une opération effectuée dans la périphérie sud-est de la cité antique permettait toutefois de restituer le plan partiel d'une villa suburbaine (Gallia, 1983 : 497-498, fig. 27 et 28). La possibilité d'intervenir en plein cœur de la ville actuelle (parking souterrain) était donc l'occasion d'établir une base de données fiables portant sur les conditions d'extension de la ville gallo-romaine et sur les solutions urbanistiques de continuité qui ont fait évoluer ou régresser le bâti jusqu'aux époques modernes.

3 Le Maubourguet est connu pour être le lieu d'implantation d'un bourg mentionné déjà entre 1260 et 1280 lors de l'installation du couvent des Cordeliers et pour recéler le tracé d'un cloaque d'époque gallo-romaine qui paraissait limiter la partie orientale de la ville antique.

4 Pour cette dernière période, les résultats de la fouille n'ont fait que confirmer les observations et hypothèses déjà évoquées : mise en évidence d'un canal (longueur repérée sur cinquante mètres, pour une largeur constante de 3,50 $\mathrm{m}$ et une élévation de 1,10 m) orienté nord-sud, fondé sur les graves et constitué de deux murs parallèles en légère inclinaison vers l'intérieur, bâtis en opusvittatum.

5 L'absence d'aménagement du radier, la structure particulière des assises supérieures constituées de plaquettes, et la largeur importante de cette structure, suggèrent un canal d'assainissement à ciel ouvert servant au captage d'un cours d'eau naturel. À l'extrémité sud de la fouille, ce canal était interrompu par une structure creuse (diamètre compris entre $3 \mathrm{~m}$ et $4 \mathrm{~m}$ ), alors qu'une déviation d'ampleur réduite (1,10 $\mathrm{m}$ de hauteur conservée, 
sur $0,75 \mathrm{~m}$ de large) venait se raccorder, côté ouest, sur le canal principal. La mise en évidence d'analogies techniques de construction indiquent un phasage qui a toutes chances d'être contemporain.

6 Le matériel archéologique recueilli - sigillées sud-gauloises des ateliers du groupe de Montans (Tarn), Drag. 22/22A, 35, 44, 29 et 37 dont une estampille probable de [N] OM [VS], sigillées hispaniques, céramiques à parois fines du type XXXV de F. Mayet, céramiques communes tournées à pâte grise (assiettes-écuelles, bols, urnes, pichet), ou claire (cruches, mortier, bol, etc.), à engobe blanc ou micacé, etc. - s'insèrent dans un contexte général qui replace l'utilisation et l'abandon de ces structures dès la période flavienne et jusqu'au tout début du II ${ }^{\mathrm{e}} \mathrm{s}$. après J.-C.

7 Un sol de béton de tuileau (épaisseur de $0,70 \mathrm{~m}$ ) a enfin été repéré à $12 \mathrm{~m}$ à l'ouest, il se place sur le même plan altimétrique que la base des murs du cloaque. Il pourrait s'agir de vestiges de bassins arasés lors de leur mise au jour en 1883. L'environnement urbain est donc extrêmement lâche et il ne fait aucun doute que nous sommes à proximité des limites de l'agglomération antique.

8 Ces résultats, qu'il conviendra de confirmer et d'intégrer aux recherches plus anciennes de R. Coquerel et de R. Vié, permettront de préciser les conditions d'implantation de l'urbanisme et son évolution architecturale qui, en d'autres points de la cité antique, évoluent entre l'époque augustéenne et l'Antiquité tardive.

9 À l'époque médiévale, la partie sud n'est qu'une succession d'apports limoneux d'origine naturelle, alors qu'au nord de la zone fouillée, se développent des aménagements structurés (niveaux de circulations et foyers) qui correspondent à des constructions légères sans doute liées à une activité artisanale. Vingt-neuf foyers ont ainsi pu être dégagés et classés selon deux indices technologiques : à simple sole d'argile ou à chape d'argile dans ou sur laquelle est venu s'ajouter un aménagement quadrangulaire complexe avec carreaux de terre cuite qui matérialisent les bordures. Leur conception est toutefois très diversifiée et une datation archéomagnétique devrait permettre de les évaluer : foyer circulaire $(0,70 \mathrm{~m}$ à $0,80 \mathrm{~m}$ de diamètre) épais de $0,05 \mathrm{~m}$ à $0,07 \mathrm{~m}$ posé sur un lit de scories et de fragments de briques et de tegulae de récupération; foyer plus élaboré avec une bordure matérialisée par un alignement de galets; foyer à plan carré où sont utilisés des fragments de briques; foyer à surface plane en carreaux de terre cuite ; foyer à carreaux de terre cuite bordé de briques verticales ; certaines surfaces de briques sont fixées au mortier de chaux.

10 La densité anormale de ces foyers, leur recharge successive qui suppose une utilisation continue et leur association à de grandes quantités de cendres (jusqu'à $0,08 \mathrm{~m}$ d'épaisseur) ne peut, d'après le fouilleur, être en rapport avec des emplacements d'habitats. Il s'agit donc de foyers établis à l'air libre dont l'un est à mettre en relation avec une série de trous de poteaux et de piquets qui délimitent une surface rectangulaire au sol d'argile rapportée et dont la bordure forme un bourrelet.

11 À proximité, d'autres trous de poteaux implantés selon des alignements plus ou moins rectilignes, matérialisent l'emplacement de structures légères qui sont considérées comme fréquemment réimplantées. Ce contexte peu structuré, s'intègre de façon logique dans une série d'aménagements de plein air et "d'habitats légers » en matériaux périssables, il est associé à des niveaux de sols aux multiples recharges, mais également à des structures bâties en creux. Il s'agit de fosses rectangulaires appuyées sur des constructions antiques préexistantes ou implantées isolément. Elles ont des volumes très 
variables $\left(2,30 \mathrm{~m}^{3}\right.$ et $\left.4,40 \mathrm{~m}^{3}\right)$ et présentent des aménagements très particuliers : orifice carré ; réutilisation des structures gallo-romaines ; doublement des parois. Une structure circulaire creuse (diamètre de $0,80 \mathrm{~m}$ ) a livré les restes de planchettes de bois assemblées qu'il est plausible d'interpréter comme les vestiges d'un tonneau partiellement enterré. Des épandages de chaux ont été reconnus alentours.

La richesse, la diversité, mais aussi la complexité de tels aménagements sont un élément de réflexion particulièrement motivant qui a conduit J.-M. Martin à une recherche plus développée. Il a pu ainsi intégrer ces types d'installations, décrits dans l'Encyclopédie de Diderot et d'Alembert, comme se rapportant à l'artisanat du cuir. Nous serions alors en présence de fosses utilisées pour le trempage des peaux, de fosses circulaires destinées à contenir le tan, alors que la chaux et les foyers sont à mettre en relation avec le traitement des peaux (passage à la flamme d'où l'utilité des foyers et épilage des peaux à l'aide de la chaux).

13 Le matériel archéologique provenant de ces milieux permet une utilisation particulièrement intéressante de la chronotypologie. Il apparaît en effet que les productions céramiques à pâte blanche (pots à liquide à col haut ou à profil bas décorés de rainures parallèles ou ondulées quelquefois entrecroisées et dont le décor s'organise parfois en chevrons et en épis, anses incisées, impressions digitales sur bourrelet rapporté, etc.) et rouge à surface griffée (pots à liquide, pots à cuire, pots à stockage, ornés d'incisions verticales barrées de traits, représentant des motifs simplifiés de palmes, de lignes verticales, de dépressions fines allongées, etc.) se retrouvent souvent en Bigorre dans des contextes compris entre le $\mathrm{XII}^{\mathrm{e}} \mathrm{s}$. et le $\mathrm{XIV}^{\mathrm{e}} \mathrm{s}$. Cela est au demeurant très significatif puisque les installations de tanneries sont mentionnées et réglementées au Maubourguet dès 1313 et jusqu'en 1340.

Dès lors l'évolution de ce quartier à vocation artisanale semble se réduire au bénéfice d'une urbanisation intensive qui se développe à partir du XIV ${ }^{\mathrm{e}} \mathrm{s}$. pour déterminer un bâti établi à partir d'un découpage parcellaire (Fig. $\mathrm{n}^{\circ} 1$ : Plan général et schématique des structures : bâtis du XVI ${ }^{\mathrm{e}} \mathrm{s}$. (en noir) et gallo-romains (en blanc) ) en lanières ( $5 \mathrm{~m}$ à $6 \mathrm{~m}$ de large) dans lequel s'organise tout un système de rues, de ruelles et de cours intérieures. Une étude architecturale précise permet, ainsi, de distinguer les murs porteurs (largeur de $0,70 \mathrm{~m}$ à $1,10 \mathrm{~m}$ ) mis en œuvre par alternance de lits de galets et de briques établis sur des fondations en fouille pleine, des murs intermédiaires de faible largeur construits à l'aide de chaux maigre qui reposent sur des sablières basses dans lesquelles s'intercalent des poteaux verticaux cloisonnant les parois de torchis. La répartition spatiale de ces deux types de construction a également provoqué la mise en évidence de critères dans les choix relatifs aux techniques de construction d'un bâti par rapport à l'autre: au nord, de part et d'autre d'une rue, utilisation du torchis, au sud, la préférence ou la condition sociale (?) a porté sur des constructions en dur. Les sols sont systématiquement en terre battue sauf, pour un cas, où un plancher sur solives a été retrouvé (planches jointives de $0,20 \mathrm{~m}$ à $0,25 \mathrm{~m}$ de large reposant sur six chevrons transversaux espacés de 1,20 m). L'incendie généralisé qui a affecté l'ensemble du quartier a provoqué la destruction totale des parties en élévation, mais a, en revanche, épargné les éléments de la couverture constituée de plaquettes d'ardoises et de tuiles canal faitières. L'arasement du bâti n'a permis de retrouver, ni les emplacements des fenêtres (mais il y a du verre à vitres), ni les seuils d'accès aux espaces extérieurs (pour une habitation, il s'agit d'un alignement de carreaux de terre cuite, bordés de montants de briques chanfreinées). 

matérialisés par des trous de calages et supports de poteaux qui cloisonnent l'habitat. La diversité des techniques mise en œuvre - série de trous de poteaux parallèles, dispositions obliques de certaines unités - conduisent à établir une systématisation des conditions de vie limitées à une pièce unique, au moins pour le rez-de-chaussée. Parmi les aménagements intérieurs particuliers, il est à noter la présence d'un four semi-enterré à usage domestique et d'une série de cuvettes aménagées dans le sol. Leur fonction est, pour la plupart, indéterminée sauf pour l'une d'entre elle sassociée à une activité métallurgique. ouest qui traverse le quartier et qui, dans son état final au XVIII ${ }^{\mathrm{s}}$., était pavée de galets avec un caniveau central en pente vers l'est, axes secondaires ou venelles non pavées (largeur évoluant entre $1 \mathrm{~m}$ et $3 \mathrm{~m}$ ) qui venaient s'appuyer contre ses bordures. Les cours intérieures délimitaient des espaces clos de grande surface (entre $35 \mathrm{~m}^{2}$ et $66 \mathrm{~m}^{2}$ ), autour desquels s'organisaient les habitats dont le système d'évacuation vers l'extérieur se déversait dans un puisard central. Le quartier du Maubourguet est, depuis l'époque galloromaine, sillonné par plusieurs fossés défensifs : fossé nord-sud au profil en « $\mathrm{V}$ » (Fig. $\mathrm{n}^{\circ}$ 2: Coupe transversale du fossé d'axe nord-sud), de $7 \mathrm{~m}$ à $8 \mathrm{~m}$ de large pour une profondeur de $5 \mathrm{~m}$, remblayé de limons très fins, d'objets de toutes sortes (nombreux restes organiques), en fonction jusqu'au $\mathrm{XVI}^{\mathrm{e}} \mathrm{s}$. et au début XVII $\mathrm{s}$.; fossé sud au profil concave d'une ouverture de $6,70 \mathrm{~m}$ pour une profondeur de $1,10 \mathrm{~m}$, bordé de part et d'autre par un ressaut, ce qui porte la largeur totale de l'ouvrage à $13,10 \mathrm{~m}$ et sa profondeur à $2,55 \mathrm{~m}$. Son remplissage est essentiellement limoneux ; une faible quantité de mobilier situe son utilisation au moins jusqu'au XVIII $\mathrm{s}$.

Les études d'archives faites conjointement ont été à l'origine d'un essai de mise en concordance historique avec les faits archéologiques. En effet, Le Terrier du début du XVI ${ }^{\mathrm{e}} \mathrm{s}$. distinguait les maisons d'habitation, mais aussi les bâtiments d'exploitation, les cours, jardins, enclos et les activités professionnelles : bouchers, cordonniers, tanneurs, tailleur, orfèvre, chaudronnier, etc. La réalité du terrain est tout autre puisque la seule attribution précise est la présence d'un atelier de dinanderie/d'orfèvrerie (louche de fondeur, peson de trébuchet, tôles de cuivre).

19 Le mobilier céramique est abondant. Les céramiques rouges communes sont dominantes, tant par le nombre que par la diversité de leurs morphologies (pots àl iquide, cruches de table, dournes, pots globulaires à une ou deux anses, pots à fond plat, couvercles, jattes évasées), alors que les décors sont inexistants, réduits semble-t-il à des incisions sur les anses. Elles sont associées à des céramiques tournées et glaçurées à pâte rouge et blanchâtre (pichets à glaçure monochrome, vases à liquide, gobelets, gargoulettes à bec tubulaire, bols à oreilles, pots à onguent), qui présentent parfois des reliefs d'appliques et un décor estampé. D'autres productions glaçurées mais polychromes sont présentes. Il s'agit le plus souvent de cruches à bec pincé, glaçurées en vert pâle sur fond blanc, en jaune vif mais également des fabrications à décors peints, dont un fragment pourrait bien provenir de Saintonge alors que d'autres céramiques présentent un décor sgraffité 
(motifs géométriques et végétaux sur des bols et écuelles). En fait, tout ceci ne semble pas original puisqu'il s'agit de formes typologiquement courantes mais dont on n'avait pu suivre, jusqu'à présent, le schéma d'évolution typochronologique, surtout pour ce secteur des Pyrénées.

Pour la première fois à Tarbes, une recherche extensive a révélé, non plus seulement, le bâti antique, mais surtout celui qui correspond aux périodes les plus proches de l'époque médiévale et post-médiévale. Cela est une nouveauté qui permet de restituer l'histoire et les faits quotidiens d'une population liée à un artisanat aujourd'hui révélé entre le XII ${ }^{\mathrm{e}} \mathrm{s}$. et le XIV ${ }^{e}$ s. Les acquis sont importants notamment sur les conditions d'évolution de la trame urbaine jusqu'à la fin du XVI ${ }^{\mathrm{e}}$ s. (1571), date à laquelle le bourg est détruit par un incendie à l'approche des troupes protestantes. Quarante ans plus tard, en 1614, les ruines sont encore visibles, puis arasées en 1619 pour faire place à une promenade.

\section{ANNEXES}

Fig. $\mathrm{n}^{\circ} 1$ : Plan général et schématique des structures : bâtis du XVI ${ }^{\mathrm{e}} \mathrm{s}$. (en noir) et gallo-romains (en blanc)

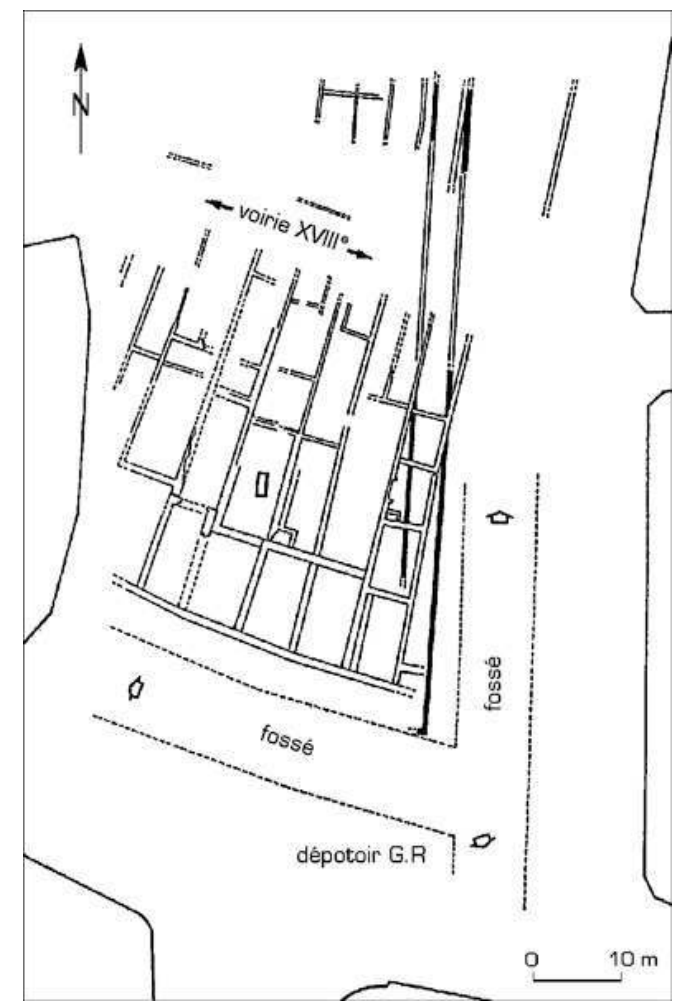

Auteur(s) : Martin, Jean-Michel. Crédits : Gl, 1997 - CNRS Editions, 1998 (1997) 
Fig. $\mathrm{n}^{\circ} 2$ : Coupe transversale du fossé d'axe nord-sud

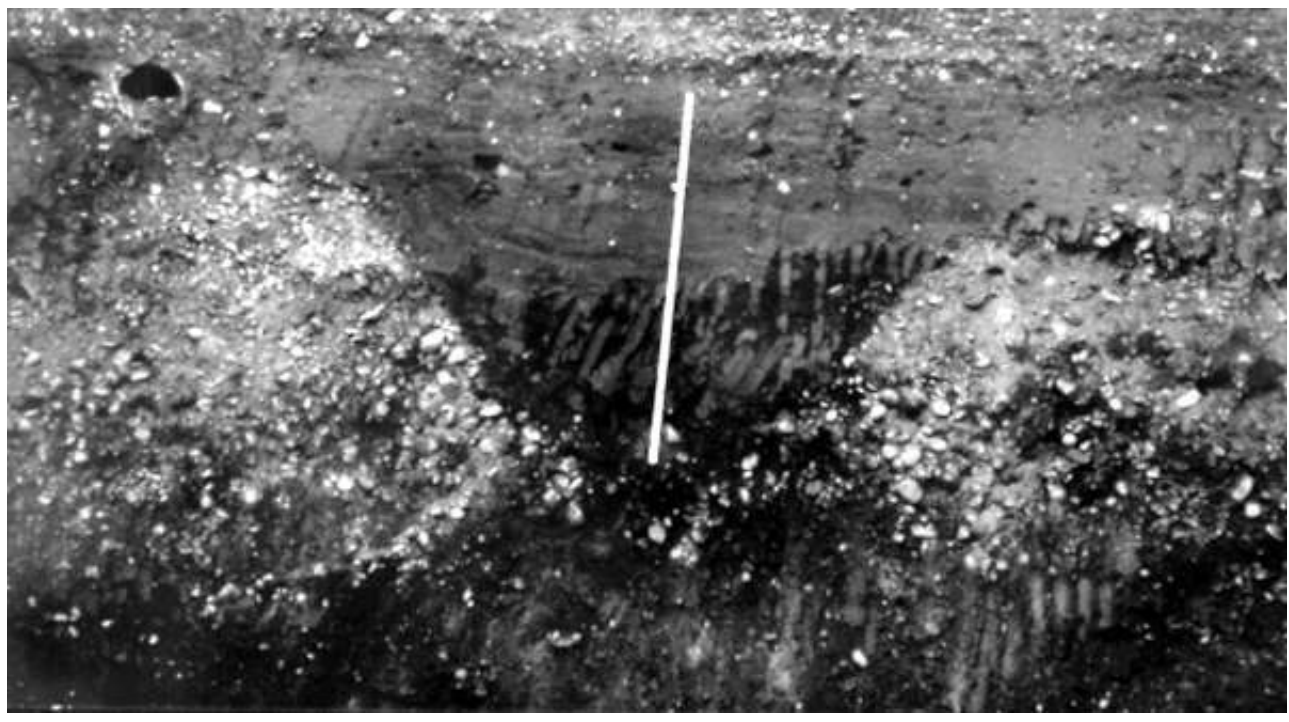

Auteur(s) : Martin, Jean-Michel. Crédits : GI, 1997 - CNRS Editions, 1998 (1997)

INDEX

Index chronologique : Antiquité romaine, Moyen Âge*, Temps Modernes Index géographique: Midi-Pyrénées, Hautes-Pyrénées (65), Tarbes operation Sauvetage programmé (SP) 